\title{
Diagnosis of coronary artery disease by radionuclide myocardial perfusion imaging
}

\author{
C Y Loong, C Anagnostopoulos
}

Heart 2004;90(Suppl V):v2-v9. doi: 10.1136/hrt.2003.013581

D espite advances in the understanding of the pathophysiology of atherosclerosis and an applaudable reduction in cardiovascular mortality over the past 30 years, coronary artery disease (CAD) remains an important cause of mortality and morbidity in the UK. While primary prevention of CAD is a priority for modern medicine, so too is the need for development of non-invasive techniques for imaging of myocardial ischaemia. Radionuclide tests occupy a central position within the cardiac imaging portfolio, and among them myocardial perfusion imaging ( $\mathrm{MPI}$ ) has an obvious place because it is the only widely available and validated method of assessing myocardial perfusion. The aim of this article is to review the literature about the role of tomographic $\mathrm{MPI}$ in the diagnosis of CAD in the general population and in specific clinical subsets, comparing it wherever possible with other imaging modalities and to summarise the recommendations from existing guidelines for the use of this technique.

\section{DIAGNOSTIC PERFORMANCE OF TOMOGRAPHIC MYOCARDIAL PERFUSION IMAGING \\ Coronary angiography and myocardial perfusion} MPI allows direct assessment of myocardial perfusion and therefore has an important role in the diagnosis of CAD in patients presenting with chest pain. An inducible perfusion abnormality indicates impaired perfusion reserve, which in turn usually corresponds to epicardial coronary obstruction. The site, depth, and extent of the abnormality provide diagnostic and management information. Conversely, a normal stress $\mathrm{MPI}$ indicates the absence of coronary obstruction and hence of clinically significant disease. A normal perfusion scan does not exclude non-obstructive CAD, but such disease is unlikely to be related to symptoms or to be prognostically important (see article on prognosis by Bateman and Prvulovich on $\mathrm{p}$ v10).

Positron emission tomography (PET) is generally considered the gold standard non-invasive technique for the assessment of myocardial perfusion. Although PET may have better accuracy than M PI, its clinical utility is constrained by high cost and poor availability. ${ }^{12}$ Therefore, MPI is the standard clinical technique for assessing myocardial perfusion, while coronary angiography is the technique for assessing epicardial coronary anatomy. Complete accuracy of $\mathrm{MPI}$ to predict the findings at angiography is neither expected nor necessary for clinical management, and when MPI is used for the diagnosis of CAD, it is not being used purely to predict the presence of epicardial coronary stenoses. Nevertheless, angiography is the accepted standard for imaging coronary arteries, and functional tests such as M PI are frequently compared with it.

\section{Sensitivity and specificity of MPI}

$M$ any studies have assessed the diagnostic accuracy of MPI for the detection of CAD, but they are of variable size and quality. Tables 1-4 summarise the findings according to the four main forms of stress; the studies ${ }^{3-64}$ are further graded for quality according to well accepted guidelines for evaluating diagnostic tests. ${ }^{65}$ The highest quality score is 3 (high), when the study involved a clearly defined population, avoided verification bias, and had independent interpretation of MPI and angiography; a quality score of 2 (medium) fulfilled two of these criteria; a score of 1 (low) fulfilled only one criterion. Because of the variation in study size, quality, and design, no attempt was made to provide weighted means of sensitivity and specificity in the above tables.

In the largest single study of 2560 patients randomised to one of the three commercial perfusion radiopharmaceuticals, and using mainly adenosine stress (the UK based ROBUST study), overall sensitivity in the subset of patients undergoing angiography was $91 \%$ and overall specificity was $87 \%$, with no significant difference between the three tracers. ${ }^{66}$ In general, review of the studies included in tables 1-4 showed that the sensitivity of MPI for detecting angiographic CAD was consistently above $70 \%$. Typical sensitivity values were in the region of $85-95 \%$ for both thallium-201(TI-201) ${ }^{6}{ }^{8}$ and technetium-99m sestamibi (sestamibi). ${ }^{17} 20$ There was a significant variation in specificity in low quality studies, ranging from $33-100 \%,{ }^{13} 14$ but carefully designed studies have reported specificities of around $75 \%$ for exercise Tl$201,{ }^{4}{ }^{10}$ and sestamibi, ${ }^{18}{ }^{19}$ and even higher values (up to 94\%) with incorporation of ECG gated data. ${ }^{67}$

For pharmacological stress performed with vasodilators, a number of good quality studies showed a sensitivity $\geqslant 90 \%$ for both TI-201 and sestamibi ${ }^{24} 27$ and a specificity $\geqslant 75 \%,{ }^{10} 2642$ with some studies reporting values as high as $100 \%{ }^{13} 2438$ For dobutamine TI-201 M PI, the sensitivity was in the region of $90 \%$ while the specificity ranged from 70 $100 \%{ }^{48}{ }^{52}$ When it was used in combination with sestamibi, most studies have shown that the sensitivity was above $80 \%,{ }^{45} 6062$ but the specificity ranged from $64-90 \%{ }^{59}{ }^{60}$

Fewer diagnostic studies have been performed using technetium-99m tetrofosmin (tetrofosmin) because of its more recent commercial introduction. For exercise tetrofosmin $\mathrm{MPI}$, the sensitivity and specificity values ranged from $81-96 \%{ }^{14}{ }^{22} 23$ and $67-91 \%,{ }^{142}$ respectively.

\section{COMPARISON OF MPI WITH NON-NUCLEAR TECHNIQUES}

Stress echocardiography

Stress echocardiography is a well validated technique for diagnosing CAD and identifies wall motion abnormalities in patients with haemodynamically significant coronary stenoses, induced by exercise or pharmacological stress. Stress induced impairment of regional endocardial excursion and of

Abbreviations: $C M R I$, cardiac magnetic resonance imaging; $C A D$, coronary artery disease; DCM, dilated cardiomyopathy; Gd-DTPA, gadolinium diethylentriaminepenta acid; $\mathrm{HCM}$, hypertrophic cardiomyopathy; LBBB, left bundle branch block; LVH, left ventricular hypertrophy; MCE, myocardial contrast echocardiography; MPI, myocardial perfusion imaging; PET, positron emission tomography; sestamibi, technetium-99m sestamibi; SPECT, single photon emission computed tomography; tetrofosmin, technetium-99m tetrofosmin; Tl201, thallium-201 
Table 1 Diagnostic performance of exercise single photon emission computed tomography (SPECT) MPI

\begin{tabular}{|c|c|c|c|c|c|c|c|}
\hline Author & $\begin{array}{l}\text { Number of } \\
\text { patients }\end{array}$ & Tracer & Analysis & MI excluded & Quality & Sensitivity & Specificity \\
\hline Tamaki $1984^{3}$ & 104 & Tl-201 & $Q$ & *both & Medium & $98 \%$ & $91 \%$ \\
\hline DePasquale $1988^{4}$ & 210 & Tl-201 & $\widehat{Q}$ & *both & Medium & $95 \%$ & $74 \%$ \\
\hline Fintel $1989^{5}$ & 135 & Tl-201 & $\mathrm{V}$ & no & Medium & $83 \%$ & - \\
\hline Iskandrian $1989^{\circ}$ & 193 & Tl-201 & V & yes & Medium & $86 \%$ & $62 \%$ \\
\hline Maddahi $1989^{7}$ & 110 & T|-201 & $Q$ & *both & High & $95 \%$ & $56 \%$ \\
\hline Mahmarian $1990^{\circ}$ & 296 & Tl-201 & $Q$ & *both & High & $87 \%$ & $87 \%$ \\
\hline Van Train $1990^{\circ}$ & 242 & Tl-201 & $\vec{Q}$ & *both & Medium & $94 \%$ & $43 \%$ \\
\hline Coyne $1991^{10}$ & 100 & Tl-201 & $\mathrm{V}$ & no & Medium & $81 \%$ & $74 \%$ \\
\hline Quinones $1992^{11}$ & 112 & Tl-201 & V & yes & Low & $77 \%$ & $81 \%$ \\
\hline Chae $1993^{12}$ & 243 & Tl-201 & $\mathrm{V}$ & no & High & $71 \%$ & $63 \%$ \\
\hline Grover-McKay $1994^{13}$ & 18 & Tl-201 & V & no & Low & $91 \%$ & $86 \%$ \\
\hline Tamaki $1994^{14}$ & 25 & Tl-201 & V & no & Low & $95 \%$ & $33 \%$ \\
\hline Ho $1997^{15}$ & 51 & Tl-201 & V & *both & High & $76 \%$ & $77 \%$ \\
\hline Kiat $1990^{16}$ & 53 & mibi & $Q$ & *both & Medium & $94 \%$ & $80 \%$ \\
\hline Pozzoli $1991^{17}$ & 75 & mibi & $\mathrm{V}$ & no & Low & $84 \%$ & $88 \%$ \\
\hline Solot $1993^{18}$ & 78 & mibi & $\mathrm{V}$ & no & Medium & $96 \%$ & $74 \%$ \\
\hline Marwick $1994^{19}$ & 86 & mibi & V & yes & Medium & $73 \%$ & $70 \%$ \\
\hline Van Train $1994^{20}$ & 124 & mibi & $Q$ & *both & Medium & $89 \%$ & $36 \%$ \\
\hline Tamaki $1994^{14}$ & 26 & tetro & $\vee$ & no & Low & $96 \%$ & $67 \%$ \\
\hline Heo $1994^{21}$ & 23 & tetro & V & no & Medium & $87 \%$ & - \\
\hline Benoit $1996^{22}$ & 30 & tetro & V & yes & Medium & $81 \%$ & $89 \%$ \\
\hline Shanoudy $1998^{23}$ & 26 & tetro & V & no & Medium & $96 \%$ & - \\
\hline
\end{tabular}

*Values were calculated for patients with and without myocardial infarction, values shown include those with myocardial infarction.

Sensitivity and specificity values for coronary stenosis $\geqslant 50 \%$. Quality is rated according to the number of quality criteria (adequate description of study group, avoidance of verification bias, avoidance of diagnostic and test review bias) met.

MI, myocardial infarction; mibi, technetium-99m sestamibi; $Q$, quantitative analysis; tetro, technetium-99m tetrofosmin; Tl-201, thallium-201; V, visual analysis.

myocardial thickening is a specific marker of myocardial ischaemia.

A recent meta-analysis by Geleijnse and Elhendy of seven studies directly comparing exercise echocardiography and exercise MPI revealed comparable sensitivities ( $78 \%$ v $83 \%$, respectively) and specificities (91\% $v 83 \%$, respectively). ${ }^{68}$ In the same review, the authors also compared dobutamine echocardiography with dobutamine $\mathrm{MPI}$; in eight studies, they found that dobutamine echocardiography had a lower sensitivity than MPI $(80 \% v 86 \%, p<0.05)$, but a higher specificity $(86 \% \quad v 73 \%$, respectively, $p<0.005) .{ }^{68}$ The sensitivity of vasodilator echocardiography, however, was significantly lower than that of vasodilator M PI ( $66 \%$ v $85 \%$, $p<0.0001$ ) in six studies. ${ }^{68}$ This is not surprising since adenosine and dipyridamole cause blood flow heterogeneity, which is useful in MPI, but generally do not result in true myocardial ischaemia, which is required for causing contractile abnormalities.
Another meta-analysis by Schinkel and colleagues examined 17 studies in which MPI was directly compared with stress echocardiography. ${ }^{69}$ Pooled data revealed that M PI was more sensitive than echocardiography (sensitivity $85 \% v 80 \%$, respectively, $p<0.05$ ) but less specific (specificity $77 \% v$ $86 \%$, respectively, $p<0.001){ }^{69}$

\section{Myocardial contrast echocardiography and cardiac magnetic resonance imaging}

A small number of studies have directly compared MPI with either myocardial contrast echocardiography (MCE) or cardiac magnetic resonance imaging (CM RI), both of which are novel techniques for the assessment of myocardial perfusion. In a small study of 30 patients who underwent dipyridamole stress testing, Kaul and colleagues found a good agreement $(\kappa=0.86)$ between $\mathrm{MPI}$ and MCE for the presence or absence of CAD. ${ }^{70} \mathrm{Wei}$ and associates performed a

Table 2 Diagnostic performance of dipyridamole SPECT MPI

\begin{tabular}{|c|c|c|c|c|c|c|c|}
\hline Author & $\begin{array}{l}\text { Number of } \\
\text { patients }\end{array}$ & Tracer & Analysis & MI excluded & Quality & Sensitivity & Specificity \\
\hline †Francisco $1982^{24}$ & 75 & Tl-201 & $Q$ & no & Medium & $90 \%$ & $96 \%$ \\
\hline †Huikuri $1988^{25}$ & 93 & Tl-201 & $\mathrm{V}$ & no & Medium & $96 \%$ & $75 \%$ \\
\hline Go $1990^{26}$ & 202 & Tl-201 & V & *both & High & $76 \%$ & $80 \%$ \\
\hline †Mendelson $1992^{27}$ & 79 & Tl-201 & V & *both & Medium & $90 \%$ & - \\
\hline Cramer $1994^{28}$ & 38 & Tl-201 & V & No & High & $90 \%$ & $71 \%$ \\
\hline Grover-McKay $1994^{13}$ & 18 & Tl-201 & V & uns & Low & $91 \%$ & $100 \%$ \\
\hline Ho $1995^{29}$ & 54 & Tl-201 & V & no & High & $98 \%$ & $73 \%$ \\
\hline Watanabe $1997^{30}$ & 53 & Tl-201 & V & yes & Medium & $80 \%$ & $72 \%$ \\
\hline Tartagni $1991^{31}$ & 30 & Tl-201; mibi & V & no & Medium & $100 \%$ & $75 \%$ \\
\hline Miller $1997^{32}$ & 244 & Mibi & V & *both & Medium & $91 \%$ & $28 \%$ \\
\hline Schillaci $1997^{33}$ & 40 & Mibi & V & yes & Low & $95 \%$ & $72 \%$ \\
\hline Soman 1997 34] & 27 & Mibi & V & no & High & $90 \%$ & $66 \%$ \\
\hline Ogilby $1998^{35}$ & 26 & Mibi & V & no & Medium & $90 \%$ & $100 \%$ \\
\hline †Santoro $1998^{36}$ & 60 & Mibi & V & yes & High & $97 \%$ & $89 \%$ \\
\hline $\mathrm{He} 1997^{37}$ & 64 & Tetro & V & no & Medium & $85 \%$ & $54 \%$ \\
\hline
\end{tabular}

*Values were calculated for patients with and without myocardial infarction, values shown include those with myocardial infarction; $\dagger$ coronary stenosis $\geqslant 70 \%$. Sensitivity and specificity values for coronary stenosis $\geqslant 50 \%$ and for coronary stenosis $\geqslant 70 \%$. Quality is rated according to the number of quality criteria (adequate description of study group, avoidance of verification bias, avoidance of diagnostic and test review bias) met.

$\mathrm{MI}$, myocardial infarction; mibi, technetium-99m sestamibi; $Q$, quantitative analysis; tetro, technetium-99m tetrofosmin; Tl-201, thallium-201; uns, unspecified; $\mathrm{V}$, visual analysis. 
Table 3 Diagnostic performance of adenosine SPECT MPI

\begin{tabular}{|c|c|c|c|c|c|c|c|}
\hline Author & $\begin{array}{l}\text { Number of } \\
\text { patients }\end{array}$ & Tracer & Analysis & MI excluded & Quality & Sensitivity & Specificity \\
\hline Nguyen $1990^{38}$ & 60 & T|-201 & $\mathrm{V}$ & no & Medium & $92 \%$ & $100 \%$ \\
\hline Verani $1990^{39}$ & 45 & T|-201 & $Q$ & no & Medium & $83 \%$ & $94 \%$ \\
\hline Coyne $1991^{10}$ & 100 & $\mathrm{~T} \mid-201$ & $\mathrm{~V}$ & *both & Medium & $83 \%$ & $76 \%$ \\
\hline Nishimura $1991^{40}$ & 101 & Tl-201 & V & *both & High & $84 \%$ & $84 \%$ \\
\hline Allman $1992^{41}$ & 76 & Tl-201 & $Q$ & yes & Low & $85 \%$ & $38 \%$ \\
\hline Pennell $1995^{42}$ & 226 & Tl-201 & $\mathrm{V}$ & no & High & $96 \%$ & $78 \%$ \\
\hline Mohiuddin $1996^{43}$ & 202 & Tl-201 & $Q$ & no & High & $90 \%$ & $86 \%$ \\
\hline Amanullah $1993^{44}$ & 40 & mibi & $\mathrm{V}$ & no & Medium & $94 \%$ & $100 \%$ \\
\hline Marwick $1993^{45}$ & 97 & mibi & V & yes & Medium & $86 \%$ & $71 \%$ \\
\hline Jamil $1999^{46}$ & 32 & mibi & V & no & Medium & $75 \%$ & - \\
\hline
\end{tabular}

*Values were calculated for patients with and without myocardial infarction, values shown include those with myocardial infarction.

Sensitivity and specificity values for coronary stenosis $\geqslant 50 \%$. Quality is rated according to the number of quality criteria (adequate description of study group, avoidance of verification bias, avoidance of diagnostic and test review bias) met.

MI, myocardial infarction; mibi, technetium-99m sestamibi; Q, quantitative analysis; tetro, technetium-99m tetrofosmin; Tl-201, thallium-201; V, visual analysis.

similar comparison in a recent multi-centre study and confirmed these results. ${ }^{71}$

CM RI has also been used for detection of CAD in a similar way to stress echocardiography; in a recent review, this technique has been shown to have a mean sensitivity and specificity of $86 \%$ and $84 \%$, respectively, for detecting CAD. ${ }^{72}$ In the last few years, direct assessment of myocardial perfusion has become feasible using gadolinium diethylentriaminepenta acid (Gd-DTPA) ${ }^{73}$ There have been no studies so far directly comparing the diagnostic accuracies of GdDTPA perfusion CMRI and MPI, and the exact role of the former in clinical practice remains to be determined.

\section{FACTORS AFFECTING OBSERVED DIAGNOSTIC PERFORMANCE OF MPI}

When the reference standard for diagnosis (in this case coronary angiography) is not used in all patients, and referral to angiography is more likely when $\mathrm{MPI}$ is abnormal, then the findings are affected by post-test referral bias and specificity appears falsely low. ${ }^{76} 77$ Indeed, if only patients with abnormal MPI undergo angiography, the observed sensitivity will be $100 \%$ and the specificity $0 \%$. The normalcy rate is therefore a better index for assessing the performance of a test in patients without disease. This is defined as the rate of normal perfusion scans in patients with a low $(<5 \%)$ likelihood of CAD based on clinical and exercise ECG features. Studies have consistently reported significantly higher normalcy rates than specificity for $\mathrm{MPI}$ in the diagnosis of CAD (table 5). The mean normalcy rate for MPI from 12 studies (543 patients) was 88\%.

Another form of bias, the pre-test referral bias, occurs when mainly high likelihood patients are referred for MPI, which increases apparent sensitivity because of the higher proportion of patients with more severe disease. ${ }^{77}{ }^{78}$ Analysis of the studies quoted in tables 1-4 showed that in the subgroup of studies where the sensitivity of exercise TI-201 MPI was re-calculated after exclusion of patients with previous myocardial infarction, mean sensitivity fell from $92 \%$ to $87 \%(p=0.008)$. Sensitivity was also higher with more extensive and severe disease, and lower with single vessel disease $(87 \% v 79 \%, p<0.0001)$ or in those with stenoses involving branches of the major vessels. Sensitivity can also be affected by the involved coronary territory; patients with single vessel disease are more likely to have an abnormal study if the lesion is in the left anterior descending than in the left circumflex artery. ${ }^{3} 29$

Table 4 Diagnostic performance of dobutamine SPECT MPI

\begin{tabular}{|c|c|c|c|c|c|c|c|}
\hline Author & Number of patients & Tracer & Analysis & MI excluded & Quality & Sensitivity & Specificity \\
\hline Pennell $1991^{47}$ & 50 & Tl-201 & V & no & Medium & $97 \%$ & $80 \%$ \\
\hline Warner $1993^{48}$ & 16 & Tl-201 & V & no & Medium & $93 \%$ & $100 \%$ \\
\hline Hays $1993^{49}$ & 67 & Tl-201 & Q & no & Medium & $86 \%$ & $90 \%$ \\
\hline Huang $1997^{50}$ & 93 & Tl-201 & $Q$ & no & High & $90 \%$ & $81 \%$ \\
\hline Huang $1998^{51}$ & 110 & Tl-201 & $\vee$ & yes & High & $82 \%$ & $82 \%$ \\
\hline Caner $1997^{52}$ & 29 & Tl-201; mibi & V & uns & High & $89 \%$ & $70 \%$ \\
\hline Gunalp $1993^{53}$ & 27 & mibi & V & yes & Medium & $94 \%$ & $89 \%$ \\
\hline Forster $1993^{54}$ & 21 & mibi & V & yes & High & $83 \%$ & $89 \%$ \\
\hline Marwick $1993^{45}$ & 97 & mibi & $\vee$ & yes & Medium & $80 \%$ & $74 \%$ \\
\hline Marwick $1993^{55}$ & 217 & mibi & $Q$ & yes & High & $76 \%$ & $67 \%$ \\
\hline Mairesse $1994^{56}$ & 129 & mibi & $\mathrm{V}$ & yes & High & $76 \%$ & $65 \%$ \\
\hline Marwick $1994^{19}$ & 82 & mibi & V & yes & Medium & $65 \%$ & $68 \%$ \\
\hline Senior $1994^{57}$ & 61 & mibi & V & no & High & $95 \%$ & $71 \%$ \\
\hline Di Bello $1996^{58}$ & 45 & mibi & V & yes & High & $87 \%$ & $86 \%$ \\
\hline Iffihar $1996^{59}$ & 38 & mibi & V & no & Medium & $79 \%$ & $90 \%$ \\
\hline Kisacik $1996^{60}$ & 69 & mibi & V & no & High & $96 \%$ & $64 \%$ \\
\hline Slavich $1996^{61}$ & 46 & mibi & V & yes & Medium & $82 \%$ & $83 \%$ \\
\hline San Roman $1998^{62}$ & 92 & mibi & $Q$ & yes & High & $87 \%$ & $70 \%$ \\
\hline †Santoro $1998^{63}$ & 60 & mibi & $\hat{Q}$ & yes & High & $91 \%$ & $81 \%$ \\
\hline Elhendy $1998^{64}$ & 70 & mibi & $\mathrm{V}$ & no & Low & $64 \%$ & $72 \%$ \\
\hline
\end{tabular}

*Values were calculated for patients with and without myocardial infarction, values shown include those with myocardial infarction; $†$ coronary stenosis $\geqslant 70 \%$. Sensitivity and specificity values for coronary stenosis $\geqslant 50 \%$ and coronary stenosis $\geqslant 70 \%$. Quality is rated according to the number of quality criteria (adequate description of study group, avoidance of verification bias, avoidance of diagnostic and test review bias) met.

MI, myocardial infarction; mibi, technetium-99m sestamibi; $Q$, quantitative analysis; tetro, technetium-99m tetrofosmin; Tl-201, thallium-201; uns, unspecified; $\mathrm{V}$, visual analysis. 
Table 5 Normalcy rates for SPECT MPI

\begin{tabular}{|c|c|c|c|c|}
\hline Author & $\begin{array}{l}\text { Number of } \\
\text { patients }\end{array}$ & Tracer & Stress & Normalcy rate \\
\hline $\begin{array}{l}\text { Iskandrian } 1989^{\circ} \\
\text { Maddahi } 1989^{7} \\
\text { Van Train } 1990^{9} \\
\text { Coyne } 1991^{10} \\
\text { Kiat } 1992^{74} \\
\text { Nishimura } 1992^{75} \\
\text { Nishimura } 1992^{75} \\
\text { Van Train } 1994^{20} \\
\text { Kiat } 1990^{16} \\
\text { Heo } 1997^{21}\end{array}$ & $\begin{array}{r}131 \\
52 \\
76 \\
45 \\
55 \\
39 \\
39 \\
37 \\
8 \\
61\end{array}$ & $\begin{array}{l}\text { Tl-201 } \\
\text { Tl-201 } \\
\text { Tl-201 } \\
\text { Tl-201 } \\
\text { Tl-201 } \\
\text { Tl-201 } \\
\text { Tl-201 } \\
\text { Tl-201 } \\
\text { mibi } \\
\text { mibi }\end{array}$ & $\begin{array}{l}\text { Exercise } \\
\text { Exercise } \\
\text { Exercise } \\
\text { Exercise, adenosine } \\
\text { Exercise } \\
\text { Exercise } \\
\text { Adenosine } \\
\text { Exercise } \\
\text { Exercise } \\
\text { Exercise }\end{array}$ & $\begin{array}{l}94 \% \\
86 \% \\
82 \% \\
80 \% \\
89 \% \\
92 \% \\
95 \% \\
81 \% \\
88 \% \\
95 \% \\
88 \% \text { mean rate }\end{array}$ \\
\hline
\end{tabular}

The intensity of stress achieved, particularly with exercise, is another factor that may influence the observed diagnostic performance of $\mathrm{MPI}$ by increasing the number of false negative results. ${ }^{6}{ }^{79}$ Anti-anginal medication can also reduce sensitivity when using exercise, and medication should ideally be discontinued before diagnostic studies. ${ }^{80}$ Furthermore, $\beta$ blockers should be discontinued for a dobutamine stress test because they attenuate the heart rate and blood pressure response and can reduce or abolish myocardial ischaemia. ${ }^{81}{ }^{82}$ The role of anti-anginal medication in vasodilator stress is less clear but one study has shown that they may reduce sensitivity. ${ }^{83}$

Tracer activity below the diaphragm is commonly seen with sestamibi and tetrofosmin, and this can reduce specificity, as seen in some studies. ${ }^{32}{ }^{37}$ Other causes of artefact that can reduce specificity are photon attenuation and scatter, patient motion, low count statistics, reconstruction errors, or processing problems. Experienced practitioners can normally identify these problems and they maintain accuracy not only by recognising common artefactual appearances, but also by taking account of the clinical circumstances. Commercially available software packages incorporate methods for recognising and correcting problems related to these factors. Almost all of these packages will also offer some form of quantitative analysis. The latter can reduce inter-observer variability but its value for accuracy is less clear, with some studies demonstrating improvement, ${ }^{3}{ }^{24}{ }^{39}$ while others not. ${ }^{4}{ }^{8}$ Correction for the undesirable effects of attenuation using simultaneous or sequential transmission imaging is also feasible but its value is not yet clearly established. ECG-gating is another option that improves accuracy and it is now used routinely in many centres. It aids the distinction between true perfusion defects and artefacts, and provides additional prognostic information from global and regional left ventricular function..$^{84}$

Despite their different physical and imaging characteristics, all three radiotracers have comparable diagnostic accuracies for detecting CAD. As technetium-99m labelled tracers have myocardial uptake curves that plateau at lower levels of hyperaemia than Tl-201, it has been suggested that they may not perform well when used with vasodilator stress. ${ }^{86}$ However, several studies with sestamibi indicate that the diagnostic accuracy is not compromised when the latter is used with adenosine or dipyridamole. ${ }^{35}{ }^{45}$ Data from studies with tetrofosmin are less consistent, with some studies reporting good accuracy with vasodilators ${ }^{66}$ and others showing it to be less sensitive than TI-201 or sestamibi for the detection of mild to moderate angiographic disease. ${ }^{87} 88$ Such underestimation has also been reported in some, but not all, studies performed with dobutamine sestamibi MPI. 6064

\section{DIAGNOSTIC PERFORMANCE OF TOMOGRAPHIC MPI IN PATIENT SUBGROUPS \\ Extensive CAD}

An important function of any non-invasive test for CAD is its ability to identify patients with extensive disease, as their prognosis is particularly poor and thus demands prompt management. It is well documented that patients with three vessel or left main stem disease almost always have an abnormal MPI study and that the sensitivity is higher in patients with multi-vessel disease compared to those with single vessel disease. ${ }^{89}$ However, the perfusion abnormality will not necessarily reflect involvement of more than one vessel; moreover, less than two thirds of such scans show the typical pattern of multi-vessel disease. ${ }^{90}$

A number of studies have observed a low sensitivity for accurately detecting three vessel disease with visual analysis. ${ }^{322}{ }^{34}$ Quantitative analysis of the washout rate in the areas of normal TI-201 uptake was found to improve the sensitivity, which can also be increased by incorporation of the clinical response to treadmill testing. ${ }^{91}$ Other scintigraphic features such as transient ischaemic dilatation and increased lung uptake of Tl-201 were found by some investigators to be useful predictors of extensive CAD. ${ }^{92} 93$

Geleijnse and Elhendy's meta-analysis of four studies (involving 220 patients) specifically investigating the accuracy of $\mathrm{MPI}$ and stress echocardiography for detecting extensive CAD found that sensitivity and specificity were comparable for both imaging modalities. ${ }^{68}$

\section{Women}

Women represent a particular group of patients in which the non-invasive diagnosis of CAD may be challenging. Exercise ECG is often used as the initial diagnostic test; however, several studies have demonstrated that it has an unacceptably high false positive rate in women. ${ }^{94} 95$ This is thought to be partly caused by the lower prevalence of CAD in women, especially in premenopausal women, and partly because the classic ECG criteria for a positive test are based on data obtained in men.

Studies examining the diagnostic performance of MPI in women suspected of having CAD have generally reported higher sensitivity and specificity values when compared with retrospective data on exercise ECG. ${ }^{67}{ }^{96} \mathrm{~A}$ recent review of seven studies involving a total of 1140 female patients reported a mean sensitivity of $78 \%$ and specificity of $86 \%$ for the diagnosis of CAD by $\mathrm{MPI}$; these values are higher than those usually obtained with exercise ECG. ${ }^{97}$ Studies which have directly compared the two modalities have also reported higher accuracy in the detection of CAD with M PI than with exercise ECG. ${ }^{98}$ On the strength of this evidence, guidelines from the British Cardiac Society/Royal College of Physicians 
suggest that in women with an intermediate probability of CAD, M PI should be the non-invasive investigation of choice for the diagnosis of CAD. ${ }^{99}$

Few studies have directly compared $\mathrm{MPI}$ and stress echocardiography in women. One study has shown no significant difference between them, ${ }^{98}$ while others have demonstrated a superior specificity of dobutamine echocardiography over dobutamine MPI in women. ${ }^{64} 100$ Based on the existing data, it seems therefore reasonable to suggest that in female patients who are unable to exercise or undergo vasodilator stress, dobutamine echocardiography is a more appropriate form of stress than M PI.

\section{Left bundle branch block (LBBB) and other ECG abnormalities}

The non-invasive detection of CAD in patients with resting LBBB poses a particular problem. Although patients with LBBB have a high prevalence of CAD, LBBB also frequently occurs in non-ischaemic cardiomyopathies. ST segment changes on exercise ECG are non-diagnostic in these patients, and abnormal septal motion associated with the conduction abnormality may reduce the diagnostic accuracy of stress/rest regional wall motion comparisons using radionuclide ventriculography or echocardiography. ${ }^{101}$

Even in the presence of angiographically normal coronary arteries, patients with LBBB often have reversible or fixed septal defects on MPI, particularly when combined with exercise or dobutamine stress. ${ }^{102} 103$ The exact mechanism is unclear but it is possible that a delayed activation of the septum in the presence of LBBB may lead to a reduction in coronary flow and hence tracer delivery to the septum. A high heart rate increases the proportion of diastolic filling that is lost, leading to a further reduction in septal coronary flow and therefore more obvious perfusion defects. When stress is performed with dipyridamole or adenosine alone it is less likely to observe such perfusion abnormalities probably because the effect of vasodilators on the heart rate and septal contraction is less than that of dynamic exercise. It has been shown that specificity with vasodilator stress improves by up to $75 \%$ and this is achieved without a reduction in sensitivity. ${ }^{104}$ Accordingly, the recently published American College of Cardiology/American Heart Association guidelines for the management of patients with stable angina recommend the use of adenosine or dipyridamole SPECT MPI as the preferred imaging modality for patients with LBBB. ${ }^{105}$ The committee does not recommend the use of echocardiography because there is only limited information regarding its clinical utility. ${ }^{106}$

As in LBBB, perfusion abnormalities may be seen in patients with bifascicular block or right ventricular pacemakers even in the absence of epicardial CAD, ${ }^{107}$ therefore if a stress test is required, this should be performed with vasodilators. M PI can also be helpful in patients with resting ECG changes (such as those seen in left ventricular hypertrophy (LVH), pre-excitation or drug effects) because they can reduce the usefulness of exercise ECG for the detection of CAD. ${ }^{105}$

\section{Cardiomyopathies and LVH}

Dilated cardiomyopathy (DCM) is often associated with perfusion abnormalities and hence the non-invasive detection of concomitant epicardial coronary stenoses can be difficult. Although a normal scan or a study showing patchy non-reversible defects in a patient with DCM virtually exclude the presence of underlying CAD as the cause of cardiomyopathy, the presence of reversible abnormalities is not always the result of CAD. ${ }^{108}$ However, if such abnormalities are found in the distribution of coronary territories, then they are more likely to represent underlying CAD and

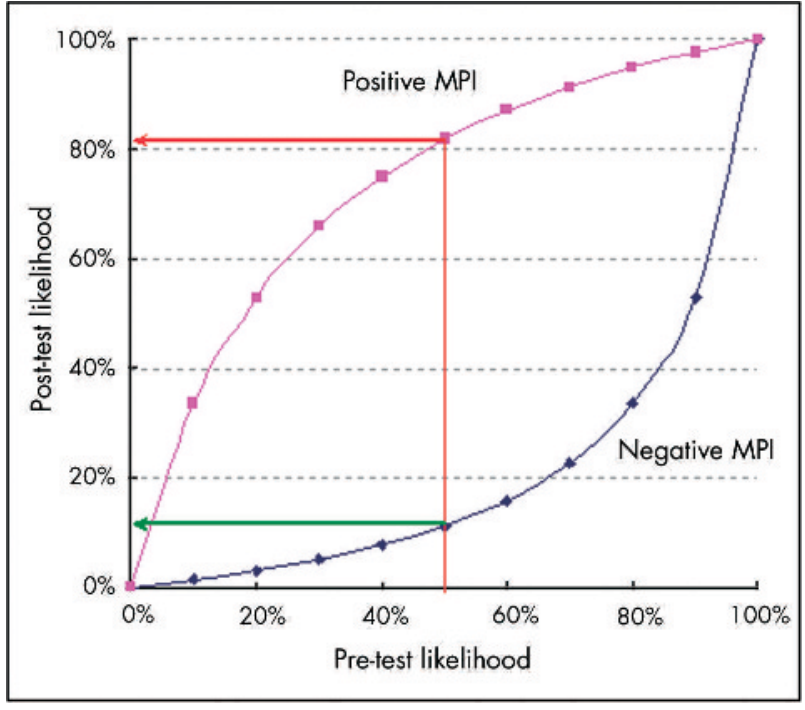

Figure 1 Sequential Bayesian analysis of CAD probability using MPI. The post-test likelihood of CAD (following MPI) can be estimated by sequential Bayesian analysis using the pre-test likelihood of disease. If MPI is positive, the post-test likelihood is calculated from the upper response line (purple), while if the test is negative, the lower response line (blue) is used. These curves are constructed for a diagnostic test (MPI) with a sensitivity of $90 \%$ and specificity of $80 \%$. For example, in a patient with a $50 \%$ pre-test likelihood of CAD, a positive MPI scan increases the post-test likelihood of disease to $81.8 \%$ (red line), while a negative scan decreases the post-test likelihood of disease to $11.1 \%$. The post-test probability difference for this patient is therefore $70.7 \%$. In contrast, for a patient with a low pre-test likelihood of disease of 5\%, a positive MPI scan increases the post-test likelihood of disease to only $19.2 \%$, while a negative scan decreases the post-test likelihood to $0.6 \%$. The post-test probability difference is therefore only $18.6 \%$.

hence, they can help to differentiate between ischaemic and non-ischaemic aetiology. This distinction is important because it has implications for the patient management (see article on myocardial viability and hibernation by Bax and colleagues on $\mathrm{p}$ v26).

Patients with hypertrophic cardiomyopathy (HCM) often demonstrate an abnormally thickened septum on MPI. However, this technique is of very limited value in the detection of the condition and confirmation of the diagnosis should be performed with echocardiography or CMRI. Perfusion abnormalities are often seen in patients with HCM in the absence of epicardial coronary disease and typically affect the septum. ${ }^{109}$ There is evidence that patients with inducible ischaemia on MPI demonstrate lactate production during rapid atrial pacing. ${ }^{110}$ This supports the view that such abnormalities represent true myocardial ischaemia, although this is presumably the result of other mechanisms, such as systolic compression of intramyocardial septal vessels, abnormal coronary flow pattern in the left anterior descending artery, or impaired coronary flow reserve. A negative perfusion scan in HCM, on the other hand, suggests that significant underlying epicardial CAD is unlikely. Inducible ischaemia on MPI has been correlated with events (cardiac arrest or syncope) in young patients with $\mathrm{HCM}^{111}$ although not in larger unselected populations. ${ }^{112}$ At present, the role of MPI in the management of patients with HCM remains undefined.

Inducible perfusion defects can also be seen in patients with more generalised LVH because of abnormalities in the microcirculation which may therefore erode the specificity of M PI for detecting epicardial CAD. The specificity of MPI may be further compromised by changes seen occasionally in the septum and lateral wall in the form of a reversal of lateral 
wall to septum tracer uptake ratio, which is not caused by CAD. ${ }^{113}$ Dobutamine echocardiography does not suffer from these limitations and although some investigators have found it to be equally effective with MPI for detecting underlying CAD ${ }^{114}$ more recent data suggest that the former is probably the technique of choice in this clinical setting. ${ }^{115}$

\section{USE OF MPI IN THE INVESTIGATIVE STRATEGIES FOR THE DIAGNOSIS OF CAD}

According to Bayes theorem, non-invasive tests are of most value in patients with an intermediate probability of CAD (fig 1). Ideally, therefore, such patients should undergo M PI without prior performance of an exercise ECG. Several factors militate against this. The most important is the relative availability of the two techniques, but radiation burden and cost are also relevant. Many centres use a staged approach with the exercise ECG being the initial test followed by MPI if the likelihood of disease is indeterminate after the exercise ECG, or if further information on myocardial perfusion is required to assist management decisions. This strategy has been adopted in recently published guidelines by the British Cardiac Society/Royal College of Physicians and European Society of Cardiology. ${ }^{99} 116$ They also recommend that MPI should be the initial investigation in patients who are unlikely to exercise adequately, in women, and in cases where the exercise ECG is uninterpretable because of resting abnormalities (see above). It is also reasonable to suggest that even patients with a high likelihood of CAD can be considered for MPI before coronary angiography. MPI can assess objectively the extent and severity of myocardial ischaemia allowing accurate risk stratification and influencing management decisions.

The role of MPI in asymptomatic patients with increased cardiovascular risk profiles is not clearly defined. The existing data suggest that MPI should not be used in screening unselected asymptomatic populations, ${ }^{117}$ but it may have a limited role in selected patients considered to be at a high risk for developing CAD (for example, men $\geqslant 45$ years old with a family history of premature CAD or patients with severe calcification on electron beam computed tomography and resting ECG abnormalities). ${ }^{118} 119$

\section{CONCLUSION}

Radionuclide M PI is a well validated, non-invasive method of assessing myocardial perfusion in men and women. It has the ability to localise haemodynamically important coronary stenoses, and assess the extent and severity of coronary obstruction. It possesses a high overall diagnostic accuracy for detecting CAD, which increases further with incorporation of ECG gated data. A normal myocardial perfusion scan makes it extremely unlikely that a patient has haemodynamically significant CAD, whatever the clinical and ECG features are, and therefore invasive investigations can be avoided in such patients.

\section{Authors' affiliations \\ C Y Loong, National Heart \& Lung Institute, Imperial College London, London, UK}

C Anagnostopoulos, Department of Nuclear Medicine, Royal Brompton Hospital, London, UK

Correspondence to: $\operatorname{Dr} C$ Anagnostopoulos, Department of Nuclear Medicine, Royal Brompton Hospital, Sydney Street, London SW3 6NP, UK; c.anagnostopoulos@imperial.ac.uk

\section{REFERENCES}

1 Go RT, Marwick TH, Maclntyre WJ, et al. A prospective comparison of rubidium-82 PET and thallium-201 SPECT myocardial perfusion imaging utilising a single dipyridamole stress in the diagnosis of coronary artery disease. J Nucl Med 1990;31:1899-905

2 Stewart R, Schwaiger M, Molina E, et al. Comparison of rubidium-82 positron emission tomography and thallium-201 SPECT imaging for detection of coronary artery disease. Am J Cardiol 1991;67:1303-10.

3 Tamaki N, Yonekura Y, Mukai T, et al. Stress thallium-201 transaxial emission computed tomography: quantitative versus qualitative analysis for evaluation of coronary artery disease. J Am Coll Cardiol 1984;4:1213-21.

4 DePasquale EE, Nody AC, DePuey EG, et al. Quantitative rotational thallium201 tomography for identifying and localizing coronary artery disease. Circulation 1988;2:316-27.

5 Fintel DJ, Links JM, Brinker JA, et al. Improved diagnostic performance of exercise thallium-201 single photon emission computed tomography over planar imaging in the diagnosis of coronary artery disease: a receiver operating characteristic analysis. J Am Coll Cardiol 1989;13:600-12.

6 Iskandrian AS, Heo J, Kong B, et al. Effect of exercise level on the ability of thallium-201 tomographic imaging in detecting coronary artery disease: analysis of 461 patients. J Am Coll Cardiol 1989;14:1477-86.

7 Maddahi J, Van Train K, Pringent F, et al. Quantitative single photon emission computed thallium-201 tomography for detection and localization of coronary artery disease: optimization and prospective validation of a new technique. J Am Coll Cardiol 1989;14:1689-99.

8 Mahmarian JJ, Boyce TM, Goldberg RK, et al. Quantitative exercise thallium201 single photon emission computed tomography for the enhanced diagnosis of ischaemic heart disease. J Am Coll Cardiol 1990;15:318-29.

9 Van Train KF, Maddahi J, Berman DS, et al. Quantitative analysis of tomographic stress thallium-201 myocardial scintigrams: a multicenter trial. J Nucl Med 1990;31:1168-79.

10 Coyne EP, Belvedere DA, Vande Streek PR, et al. Thallium-201 scintigraphy after intravenous infusion of adenosine compared with exercise thallium testing in the diagnosis of coronary artery disease. J Am Coll Cardiol 1991;17:1289-94.

11 Quinones MA, Verani MS, Haichin RM, et al. Exercise echocardiography versus Tl-201 single-photon emission computed tomography in evaluation of coronary artery disease. Circulation 1992;85:1026-31.

12 Chae SC, Heo J, Iskandrian AS, et al. Identification of extensive coronary artery disease in women by exercise single-photon emission computed tomographic (SPECT) thallium imaging. J Am Coll Cardiol 1993;21:1305-11.

13 Grover-McKay M, Milne N, Atwood E, et al. Comparison of thallium-201 single-photon emission computed tomographic scintigraphy with intravenous dipyridamole and arm exercise. Am Heart J 1994; 127:1516-20.

14 Tamaki N, Takahashi N, Kawamoto M, et al. Myocardial tomography using technetium-99m tetrofosmin to evaluate coronary artery disease. J Nucl Med 1994;35:594-600.

15 Ho Y-L, Wu C-C, Huang P-J, et al. Dobutamine stress echocardiography compared with exercise thallium-201 single-photon emission computed tomography in detecting coronary artery disease - effect of exercise level on accuracy. Cardiol 1997;88:379-85.

16 Kiat H, Van Train KF, Maddahi J, et al. Development and prospective application of quantitative 2-day stress-rest Tc-99m methoxy isobutyl isonitrile SPECT for the diagnosis of coronary artery disease. Am Heart $J$ 1990;120:1255-66

17 Pozzoli MMA, Fioretti P, Salustri A, et al. Exercise echocardiography and technetium-99m MIBI single-photon emission computed tomography in the detection of coronary artery disease. Am J Cardiol 1991;67:350-5.

18 Solot G, Hermans J, Merlo P, et al. Correlation of Tc-99m sestamibi SPECT with coronary angiography in general hospital practice. Nucl Med Commun 1993;14:23-9.

19 Marwick TH, D'Hondt AM, Mairesse GH, et al. Comparative ability of dobutamine and exercise stress in inducing myocardial ischaemia in active patients. Br Heart J 1994;72:31-8.

20 Van Train KF, Garcia EV, Maddahi J, et al. Multicenter trial validation for quantitative analysis of same-day rest-stress technetium-99m-sestamibi myocardial tomograms. J Nucl Med 1994;35:609-18.

21 Heo J, Cave V, Wasserleben V, et al. Planar and tomographic imaging with technetium-99m-labeled tetrofosmin: correlation with thallium-201 and coronary angiography. J Nucl Cardiol 1994;1:317-24.

22 Benoit Th, Vivegnis D, Lahiri A, et al. Tomographic myocardial imaging with technetium-99m tetrofosmin. Eur Heart J 1996; 17:635-42.

23 Shanoudy H, Raggi P, Beller GA, et al. Comparison of technetium-99m tetrofosmin and thallium-201 single-photon emission computed tomographic imaging for detection of myocardial perfusion defects in patients with coronary artery disease. J Am Coll Cardiol 1998:31:331-7.

24 Francisco DA, Collins SM, Go RT, et al. Tomographic thaalium-201 myocardial perfusion scintigrams after maximal coronary artery vasodilation with intravenous dipyridamole. Circulation 1982;66:370-9.

25 Huikuri HV, Korhonen UR, Airaksinen KEJ, et al. Comparison of dipyridamole-handgrip test and bicycle exercise test for thallium tomographic imaging. Am J Cardiol 1988;61:264-8.

26 Go RT, Marwick TH, Maclntyre WJ, et al. A prospective comparison of rubidium-82 PET and thallium-201 SPECT myocardial perfusion imaging utilizing a single dipyridamole stress in the diagnosis of coronary artery disease. J Nucl Med 1990;31:1899-905.

27 Mendelson MA, Spies SM, Spies WG, et al. Usefulness of single-photon emission computed tomography of thallium-201 uptake after dipyridamole infusion for detection of coronary artery disease. Am J Cardiol 1992;69: 11 150-5

28 Cramer $M-J$, Verzijlbergen JF, Van der Wall EE, et al. Head-to-head comparison between technetium-99m-sestamibi and thallium-201 
tomographic imaging for the detection of coronary artery disease using combined dipyridamole-exercise stress. Coron Artery Dis 1994;5:787-91.

29 Ho F-M, Huang P-J, Liau C-S, et al. Dobutamine stress echocardiography compared with dipyridamole thallium-201 single-photon emission computed tomography in detecting coronary artery disease. Eur Heart $J$ 1995; 16:570-5.

30 Watanabe K, Sekiya M, lkeda S, et al. Comparison of adenosine triphosphate and dipyridamole in diagnosis by thallium-201 myocardial scintigraphy. J Nucl Med 1997:38:577-81.

31 Tartagni F, Dondi $M$, Limonetti $P$, et al. Dipyridamole technetium- $99 \mathrm{~m} 2$ methoxy isobutyl isonitrile tomoscintigraphic imaging for identifying diseased coronary vessels: comparison with thallium-201 stress-rest study. J Nucl Med 1991;32:369-76.

32 Miller DD, Younis LT, Chaitman BR, et al. Diagnostic accuracy of dipyridamole technetium-99m-labeled sestamibi myocardial tomography for detection of coronary artery disease. J Nucl Cardiol 1997;4:18-24.

33 Schillaci O, Moroni C, Scopinaro F, et al. Technetium- $99 \mathrm{~m}$ sestamibi myocardial tomography based on dipyridamole echocardiography testing in hypertensive patients with chest pain. Eur I Nucl Med 1997;24:774-8.

34 Soman P, Khattar R, Senior R, et al. Inotropic stress with arbutamine is superior to vasodilator stress with dipyridamole for the detection of reversible ischaemia with Tc-99m sestamibi single-photon emission computed tomography. J Nucl Cardiol 1997;4:364-71.

35 Ogilby JD, Kegel JG, Heo J, et al. Correlation between haemodynamic changes and tomographic sestamibi imaging during dipyridamole-induced coronary hyperaemia. J Am Coll Cardiol 1998:31:75-82.

36 Santoro GM, Sciagra R, Buonamici P, et al. Head-to-head comparison of exercise stress testing, pharmacologic stress echocardiography and perfusion tomography as first-line examination for chest pain in patients without history of coronary artery disease. J Nucl Cardiol 1998:5:19-27.

$37 \mathrm{He}$ Z-X, Iskandrian AS, Gupta NC, et al. Assessing coronary artery disease with dipyridamole technetium-99m tetrofosmin SPECT: a multicenter trial. J Nucl Med 1997;38:44-8.

38 Nguyen T, Heo J, Ogilby JD, et al. Single photon emission computed tomography with thallium-201 during adenosine-induced coronary hyperaemia: correlation with coronary arteriography, exercise thallium imaging and two-dimensional echocardiography. J Am Coll Cardiol 1990;16:1375-83.

39 Verani MS, Mahmarian JJ, Hixson JB, et al. Diagnosis of coronary artery disease by controlled coronary vasodilation with adenosine and thallium201 scintigraphy in patients unable to exercise. Circulation 1990;82:80-7

40 Nishimura S, Mahmarian JJ, Boyce TM, et al. Quantitative thallium-201 single-photon emission computed tomography during maximal pharmacologic coronary vasodilation with adenosine for assessing coronary artery disease. J Am Coll Cardiol 1991;18:736-45.

41 Allman KC, Berry J, Sucharski LA, et al. Determination of extent and location of coronary artery disease in patients without prior myocardial infarction by thallium-201 tomography with pharmacologic stress. J Nucl Med 1992;33:2067-73.

42 Pennell DJ, Mavrogeni SI, Forbat SM, et al. Adenosine combined with dynamic exercise for myocardial perfusion imaging. I Am Coll Cardiol 1995:25:1300-9.

43 Mohiuddin SM, Ravage CK, Esterbrooks DJ, et al. The comparative safety and diagnostic accuracy of adenosine myocardial perfusion imaging in women versus men. Pharmacotherapy 1996;16:646-51.

44 Amanullah AM, Bevegard S, Lindvall K, et al. Assessment of left ventricular wall motion in angina pectoris by two-dimensional echocardiography and myocardial perfusion by technetium- $99 \mathrm{~m}$ sestamibi tomography during adenosine-induced coronary vasodilation and comparison with coronary angiography. Am J Cardiol 1993;72:983-9.

45 Marwick T, Willemart B, D'Hondt A-M, et al. Selection of the optimal nonexercise stress for the evaluation of ischaemic regional myocardial dysfunction and malperfusion. Circulation 1993;87:345-54.

46 Jamil G, Ahlberg AW, Elliott MD, et al. Impact of limited treadmill exercise on adenosine Tc-99m sestamibi single-photon emission computed tomographic myocardial perfusion imaging in coronary artery disease. Am J Cardiol 1999;84:400-3.

47 Pennell DJ, Underwood SR, Swanton RH, et al. Dobutamine thallium myocardial perfusion tomography. J Am Coll Cardiol 1991;18:1471-9.

48 Warner MF, Pippin JJ, DiSciascio G, et al. Assessment of thallium scintigraphy and echocardiography during dobutamine infusion for the detection of coronary artery disease. Cathet Cardiovasc Diagn 1993:29:122-7.

49 Hays JT, Mahmarian JJ, Cochran AJ, et al. Dobutamine thallium-201 tomography for evaluating patients with suspected coronary artery disease unable to undergo exercise or vasodilator pharmacologic stress testing. J Am Coll Cardiol 1993;21:1583-90.

50 Huang P-J, Ho Y-L, Wu C-C, et al. Simultaneous dobutamine stress echocardiography and thallium-201 perfusion imaging for the detection of coronary artery disease. Cardiology 1997;88:556-62.

51 Huang P-J, Yen R-F, Chieng P-U, et al. Do B-blockers affect the diagnostic sensitivity of dobutamine stress thallium-201 single photon emission computed tomographic imaging? I Nucl Cardiol 1998;5:34-9.

52 Caner B, Karanfil A, Uysal U, et al. Effect of an additional atropine injection during dobutamine infusion for myocardial SPET. Nucl Med Comm 1997; 18:567-73.

53 Gunalp B, Dokumaci B, Uyan C, et al. Value of dobutamine technetium- $99 \mathrm{~m}$ sestamibi SPECT and echocardiography in the detection of coronary artery disease compared with coronary angiography. J Nucl Med 1993;34:889-94.
54 Forster T, McNeill AJ, Salustri A, et al. Simultaneous dobutamine stress echocardiography and technetium- $99 \mathrm{~m}$ isonitrile single-photon emission computed tomography in patients with suspected coronary artery disease. $J$ Am Coll Cardiol 1993;21:1591-6.

55 Marwick T, D'Hondt A-M, Baudhuin T, et al. Optimal use of dobutamine stress for the detection and evaluation of coronary artery disease: combination with echocardiography or scintigraphy, or both? J Am Coll Cardiol 1993;22:159-67.

56 Mairesse GH, Marwick TH, Vanoverschelde J-L, et al. How accurate is dobutamine stress electrocardiography for detection of coronary artery disease? Comparison with two-dimensional echocardiography and technetium-99m methoxy isobutyl isonitrile perfusion scintigraphy. J Am Coll Cardiol 1994:24:920-7.

57 Senior R, Sridhara BS, Anagnostou E, et al. Synergistic value of simultaneous stress dobutamine sestamibi single-photon emission computerized tomography and echocardiography in the detection of coronary artery disease. Am Heart J 1994;128:713-8.

58 Di Bello V, Bellina CR, Gori E, et al. Incremental diagnostic value of dobutamine stress echocardiography and dobutamine scintigraphy (technetium-99m-labeled sestamibi single-photon emission computed tomography) for assessment of presence and extent of coronary artery disease. J Nucl Cardiol 1996:3:212-20.

59 Iftihar I, Koutelou M, Mahmarian JJ, et al. Simultaneous perfusion tomography and radionuclide angiography during dobutamine stress. J Nucl Med 1996;37:1306-10.

60 Kisacik HL, Ozdemir K, Altinyay E, et al. Comparison of exercise stress testing with simultaneous dobutamine stress echocardiography and technetium-99m isonitrile single-photon emission computerized tomography for diagnosis of coronary artery disease. Eur Heart $J$ 1996;17:113-9.

61 Slavich GA, Guerra UP, Morocutti G, et al. Feasibility of simultaneous Tc$99 \mathrm{~m}$ sestamibi and $2 \mathrm{D}$-echo cardiac imaging during dobutamine pharmacologic stress: preliminary results in a female population. Int I Card Imaging 1996;12:113-8.

62 San Roman JA, Vilacosta I, Castillo JA, et al. Selection of the optimal stress test for the diagnosis of coronary artery disease. Heart 1998:80:370-6.

63 Santoro GM, Sciagra R, Buonamici P, et al. Head-to-head comparison of exercise stress testing, pharmacologic stress echocardiography and perfusion tomography as first-line examination for chest pain in patients without history of coronary artery disease. J Nucl Cardiol 1998:5:19-27.

64 Elhendy A, von Domburg RT, Bax JJ, et al. Non-invasive diagnosis of coronary artery stenosis in women with limited exercise capacity: comparison of dobutamine stress echocardiography and Tc-99m sestamibi single-photon emission CT. Chest 1998;114:1097-104.

65 Irwig L, Tosteson ANA, Gatsonis C, et al. Guidelines for meta-analyses evaluating diagnostic tests. Ann Intern Med 1994;120:667-76.

66 Kapur A, Latus KA, Davies G, et al. A comparison of three radionuclide myocardial perfusion tracers in clinical practice: the ROBUST study. Eur JNud Med 2002;29:1608-16.

67 Taillefer R, DePuey G, Udelson JE, et al. Comparative diagnostic accuracy of TI-201 and Tc-99m sestamibi SPECT imaging (perfusion and ECG-gated SPECT) in detecting coronary artery disease in women. J Am Coll Cardiol 1997:29:69-77.

68 Geleijnse ML, Elhendy A. Can stress echocardiography compete with perfusion scintigraphy in the detection of coronary artery disease and cardiac risk assessment? Eur J Echocardiogr 2000;1:12-21.

69 Schinkel AFL, Bax JJ, Geleiinse ML, et al. Noninvasive evaluation of ischaemic heart disease: myocardial perfusion imaging or stress echocardiography? Eur Heart J 2003;24:789-800.

70 Kaul S, Senior R, Dittrich H, et al. Detection of coronary artery disease with myocardial contrast echocardiography: comparison with Tc-99m sestamibi single-photon emission computed tomography. Circulation 1997:96:785-92.

71 Wei K, Crouse L, Weiss J, et al. Comparison of usefulness of dipyridamole stress myocardial contrast echocardiography to technetium- $99 \mathrm{~m}$ sestamibi single-photon emission computed tomography for detection of coronary artery disease (PB127 multicenter phase 2 trial results). Am J Cardiol 2003;91:1293-8.

72 Pennell DJ. Stress CMR-wall motion. In: Manning WJ, Pennell DJ, eds. Cardiovascular magnetic resonance. Philadelphia: Churchill Livingstone, 2002:120-4.

73 Muhling O, Jerosch-Herold M, Nabauer M, et al. Assessment of ischaemic heart disease using magnetic resonance first-pass perfusion imaging. Herz 2003;28:82-9.

74 Kiat $\mathrm{H}$, Van Train KF, Friedman JD, et al. Quantitative stress-redistribution thallium-201 SPECT using prone imaging: methodologic development and validation. J Nucl Med 1992;33:1509-15.

75 Nishimura S, Mahmarian JJ, Boyce TM, et al. Equivalence between adenosine and exercise thallium-201 myocardial tomography: a multicenter, prospective, crossover trial. J Am Coll Cardiol 1992;20:265-75.

76 Begg CB, Greenes RA. Assessment of diagnostic tests when disease verification is subject to selection bias. Biometrics 1983;39:207-15.

77 Rozanski A. Referral bias and the efficacy of radionuclide stress tests: problems and solutions. J Nucl Med 1992;33:2074-9.

78 Detrano R, Janosi A, Lyons KP, et al. Factors affecting sensitivity and specificity of a diagnostic test: the exercise thallium scintigram. Am J Med 1988;84:699-710.

79 Heller GV, Ahmed I, Tilkemeier PL, et al. Comparison of chest pain, electrocardiographic changes and thallium-201 scintigraphy during varying exercise intensities in men with stable angina pectoris. Am J Cardiol 1991;68:569-74. 
80 Hockings B, Saltissi S, Croft DN, et al. Effect of beta adrenergic blockade on thallium-201 myocardial perfusion imaging. Br Heart J 1983;49:83-9.

81 Shehata AR, Gillam LD, Mascitelli VA, et al. Impact of acute propanolol administration on dobutamine-induced myocardial ischaemia as evaluated by myocardial perfusion imaging and echocardiography. Am J Cardiol 1997;80:268-72.

82 Murthy DR, White CM, Katten D, et al. Effect of intravenous metoprolol or intravenous metoprolol plus glucagons on dobutamine-induced myocardia ischaemia. Pharmacotherapy 2000;20:1303-9.

83 Sharir T, Rabinowitz B, Livschitz S, et al. Underestimation of extent and severity of coronary artery disease by dipyridamole stress thallium-201 single-photon emission computed tomographic myocardial perfusion imaging in patients taking antianginal drugs. J Am Coll Cardio 1998;31:1540-6.

84 Smanio PE, Watson DD, Segalla DL, et al. Value of gating of technetium$99 \mathrm{~m}$ sestamibi single-photon emission computed tomographic imaging. J Am Coll Cardiol 1997;30:1687-92.

85 Sharir T, Germano G, Kavanagh PB, et al. Incremental prognostic value of post-stress left ventricular ejection fraction and volume by gated myocardial perfusion single photon emission computed tomography. Circulation 1999; 100:1035-42.

86 Glover DK, Ruiz M, Yang JY, et al. Myocardial Tc- $99 \mathrm{~m}$ tetrofosmin uptake during adenosine-induced vasodilatation with either a critical or mild coronary stenosis: comparison with $\mathrm{Tl}-201$ and regional myocardial blood flow. Circulation 1997; $96: 2332-8$

87 Soman O, Taillefer R, dePuey EG, et al. Enhanced detection of reversible perfusion defects by Tc-99m sestamibi compared to Tc-99m tetrofosmin during vasodilator stress SPECT imaging in mild-to-moderate coronary artery disease. J Am Coll Cardiol 2001;37:458-62.

88 Shanoudy H, Raggi P, Beller GA, et al. Comparison of technetium- $99 \mathrm{~m}$ tetrofosmin and thallium-201 single-photon emission computed tomographic imaging for detection of myocardial perfusion defects in patients with coronary artery disease. J Am Coll Cardiol 1998;31:331-7.

89 Dash H, Massie BM, Botvinick EH, et al. The noninvasive identification of left main and three-vessel coronary artery disease by myocardial stress perfusion scintigraphy and treadmill exercise electrocardiography. Circulation 1979;60:276-84.

90 Udelson JE, Rajendran V, Leppo JA. Diagnosis of coronary artery disease. In: Murray IPC, Ell PJ, eds. Nuclear medicine in clinical diagnosis and treatment, volume 2, 2nd ed. London: Churchill Livingstone, 1998:1389-413

91 Kwok JMF, Christian TF, Miller TD, et al. Identification of severe coronary artery disease in patients with a single abnormal coronary territory on exercise thallium-201 imaging: the importance of clinical and exercise variable. J Am Coll Cardiol 2000;35:335-44.

92 Daou D, Delahaye N, Lebtahi R, et al. Diagnosis of extensive coronary artery disease: intrinsic value of increased lung Tl-201 uptake with exercise SPECT. J Nucl Med 2000:41:567-74.

93 Ho K-T, Miller TD, Christian TF, et al. Prediction of severe coronary artery disease and long-term outcome in patients undergoing vasodilator SPECT. J Nucl Cardiol 2001;8:438-44.

94 Detry JM, Kapita BM, Cosyms J, et al. Diagnostic value of history and maximal exercise electrocardiography in men and women suspected on coronary heart disease. Circulation 1977;55:756-61.

95 Guiteras $\mathrm{P}$, Chaitman BR, Waters DD, et al. Diagnostic accuracy of exercise ECG lead systems in clinical subsets of women. Circulation 1982;65:1465-74.

96 Amanullah AM, Kiat H, Friedman JD, Berman DS. Adenosine technetium$99 \mathrm{~m}$ sestamibi myocardial perfusion SPECT in women: diagnostic accuracy in detection of coronary artery disease. J Am Coll Cardiol 1996;27:803-9.

97 Iskandrian AE, Heo J, Nallamothu N. Detection of coronary artery disease in women with use of stress single-photon emission computed tomography myocardial perfusion imaging. J Nucl Cardiol 1997;4:329-35

98 Ho Y-L, Wu C-C, Lin L-C, et al. Assessment of coronary artery disease in women by dobutamine stress echocardiography: comparison with stress thallium-201 single-photon emission computed tomography and exercise electrocardiography. Am Heart J 1998;135:655-62.

99 de Bono D. Investigation and management of stable angina: revised guidelines 1998. Heart 1999;81:546-55.
100 Takeuchi M, Sonoda S, Miura Y, et al. Comparative diagnostic value of dobutamine stress echocardiography and stress thallium-201 single-photon emission computed tomography for detecting coronary artery disease in women. Coron Artery Dis 1996;7:831-5.

101 Bramlet DA Morris KG, Coleman RE, et al. Effect of rate-dependent left bundle branch block on global and regional left ventricular function. Circulation 1983;67:1059-65.

102 Braat SH, Brugada P, Bar FW, et al. Thallium-201 exercise scintigraphy and left bundle branch block. Am J Cardiol 1984:55:224-6.

103 Caner B, Rezaghi C, Uysal U, et al. Dobutamine thallium-201 myocardial SPECT in patients with left bundle branch block and normal coronary arteries. J Nud Med 1997;38:424-7.

104 Ebersole DG, Heironimus J, Toney MO, et al. Comparison of exercise and adenosine technetium-99m sestamibi myocardial scintigraphy for diagnosis of coronary artery disease in patients with left bundle branch block. Am J Cardiol 1993;71:450-3.

105 Gibbons RJ, Balady GJ, Bricker JT, et al. ACC/AHA 2002 guidelines update for exercise testing. American College of Cardiology website: www.acc.org/ clinical/guidelines/exercise/dirlndex.htm.

106 Vigna C, Stanislao M, De Rito V, et al. Dipyridamole stress echocardiography vs. dipyridamole sestamibi scintigraphy for diagnosing coronary artery disease in left bundle branch block. Chest 2001:120:1534-9

107 Lakkis NM, He Z-H, Verani MS. Diagnosis of coronary artery disease by exercise thallium-201 tomography in patients with a right ventricular pacemaker. J Am Coll Cardiol 1997;29:1221-5.

108 Iskandrian AS, Hakki A-H, Kane S. Resting thallium-201 myocardial perfusion patterns in patients with severe left ventricular dysfunction: differences between patients with primary cardiomyopathy, chronic coronary artery disease or acute myocardial infarction. Am Heart $J$ $1986 ; 111: 760-7$.

109 O'Gara PJ, Bonow RO, Maron BJ, et al. Myocardial perfusion abnormalities in patients with hypertrophic cardiomyopathy: assessment with thallium-201 emission computed tomography. Circulation 1987;76:1214-23.

110 Cannon RO III, Dilsizian V, O'Gara PT, et al. Myocardial metabolic, haemodynamic and electrocardiographic significance of reversible thallium201 abnormalities in hypertrophic cardiomyopathy. Circulation 1991:83:1660-7.

111 Dilsizian V, Bonow RO, Epstein SE, Fananapazir L. Myocardial ischaemia detected by thallium scintigraphy is frequently related to cardiac arrest and syncope in young patients with hypertrophic cardiomyopathy. J Am Coll Cardiol 1993;22:796-804

112 Yamada M, Elliott PM, Kaski JC, et al. Dipyridamole stress thallium-201 perfusion abnormalities in patients with hypertrophic cardiomyopathy: relationship to clinical presentation and outcome. Eur Heart $J$ 1998;19:500-7

113 Tubau JF, Szlachcic J, Hollenberg M, et al. Usefulness of thallium-201 scintigraphy in predicting the development of angina pectoris in hypertensive patients with left ventricular hypertrophy. Am J Cardiol 1989;64:45-9.

114 Elhendy A, Geleijnse ML, van Domburg RT, et al. Comparison of dobutamine stress echocardiography and technetium- $99 \mathrm{~m}$ sestamibi singlephoton emission tomography for the diagnosis of coronary artery disease in hypertensive patients with and without left ventricular hypertrophy. Eur J Nucl Med 1998;25:69-78.

115 Fragasso G, Lu C, Dabrowski P, et al. Comparison of stress/rest myocardial perfusion tomography, dipyridamole and dobutamine stress echocardiography for the detection of coronary disease in hypertensive patients with chest pain and positive exercise test. J Am Coll Cardiol 1999;34:441-7.

116 Task Force of the European Society of Cardiology. Management of stable angina pectoris. Eur Heart J 1997; 18:394-413.

117 Schwartz RS, Jackson WG, Celio PV, et al. Accuracy of exercise Tl-201 myocardial scintigraphy in asymptomatic young men. Circulation 1993;87: 165-72.

118 Blumenthal RS, Becker DM, Moy TF, et al. Exercise thallium tomography predicts future clinically manifest coronary heart disease in a high-risk asymptomatic population. Circulation 1996;93:915-23.

119 Berman DS, Schisterman EF, Miranda R, et al. Nuclear cardiology and electron-beam computed tomography: competitive or complementary? Am J Cardiol 2001;88(2A):51-5E. 